\title{
What is the physiological time to recovery after concussion? A systematic review
}

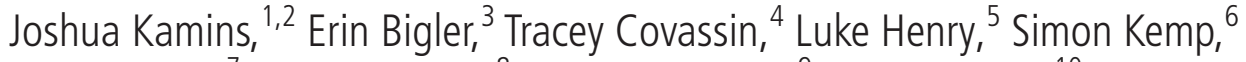 \\ John J Leddy, ${ }^{7}$ Andrew Mayer, ${ }^{8}$ Michael McCrea, ${ }^{9}$ Mayumi Prins, ${ }^{10}$ \\ Kathryn J Schneider, ${ }^{11}$ Tamara C Valovich McLeod, ${ }^{12}$ Roger Zemek, ${ }^{13}$ \\ Christopher C Giza1,2,14
}

\begin{abstract}
- Additional material is published online only. To view please visit the journal online (http://dx.doi.org/10.1136/ bjsports-2016-097464).
\end{abstract}

For numbered affiliations see end of article.

Correspondence to Dr Christopher C Giza, Department of Neurosurgery, UCLA Steve Tisch BrainSPORT Program, Room 531 Wasserman, 300 Stein Plaza, Los Angeles 90095, California, USA; cgiza@mednet.ucla.edu

Accepted 22 March 2017 Published Online First 28 March 2017

\section{ABSTRACT}

Aim or objective The aim of this study is to consolidate studies of physiological measures following sport-related concussion (SRC) to determine if a time course of postinjury altered neurobiology can be outlined. This biological time course was considered with respect to clinically relevant outcomes such as vulnerability to repeat injury and safe timing of return to physical contact risk.

Design Systematic review.

Data sources PubMed, CINAHL, Cochrane Central, PsychINFO.

Eligibility criteria for selecting studies Studies were included if they reported original research on physiological or neurobiological changes after SRC. Excluded were cases series $<5$ subjects, reviews, metaanalyses, editorials, animal research and research not pertaining to SRC.

Results A total of 5834 articles were identified, of which 80 were included for full-text data extraction and review. Relatively few longitudinal studies exist that follow both physiological dysfunction and clinical measures to recovery.

Summary/conclusions Modalities of measuring physiological change after SRC were categorised into the following: functional MRI, diffusion tensor imaging, magnetic resonance spectroscopy, cerebral blood flow, electrophysiology, heart rate, exercise, fluid biomarkers and transcranial magnetic stimulation. Due to differences in modalities, time course, study design and outcomes, it is not possible to define a single 'physiological time window' for SRC recovery. Multiple studies suggest physiological dysfunction may outlast current clinical measures of recovery, supporting a buffer zone of gradually increasing activity before full contact risk. Future studies need to use generalisable populations, longitudinal designs following to physiological and clinical recovery and careful correlation of neurobiological modalities with clinical measures.

\section{INTRODUCTION}

Establishing the time of recovery after a concussion is a difficult task for healthcare providers. These determinations have been limited by subjecCrossMark To cite: Kamins J, Bigler E, Covassin T, et al. Br J Sports Med 2017:51:939-940. tive symptom scores and imperfect clinical and neuropsychological testing. In addition, patients frequently experience more persistent symptoms including, but not limited to, chronic migraines, anxiety, post-traumatic stress disorder, attention problems and sleep dysfunction. Clinicians must determine whether these are premorbid maladies, downstream effects of concussion, unrelated phenomena or a true representation of ongoing concussion pathophysiology. Because of these assessment challenges and the potential for repeat injuries when returning patients to sports too early, clinicians are frequently left in a quandary with limited data to guide decision-making. Some recent studies suggest the physiological time of recovery may outlast the time for clinical recovery. ${ }^{1-3}$ The consequences of this are uncertain, but one possibility is that athletes may be exposed to additional risk by returning to play while there is ongoing brain dysfunction. Possible hazards may include repeat injury, prolonged symptoms, increased risk of musculoskeletal injury, more severe physiological dysfunction or increased risk for neurodegenerative disease. $^{4-7}$

Therefore, this systematic review addressed three three main clinical questions:

1. How does the time course of physiological recovery compare with the time line of clinical recovery after sport-related concussion (SRC)?

2. Should there be a minimum stand-down period following SRC?

3. Is the duration or content of graded return to play progressions appropriate?

\section{METHODS}

To identify relevant articles, we searched PubMed, CINAHL, Cochrane Central and PsychINFO. In order not to miss potentially relevant studies and because of the broad range of physiological markers, our search strategy included five search categories (exercise tolerance, brain physiology, advanced brain imaging, blood/serum biomarkers and functional assessment of physiology) (table 1). The initial search, designed in collaboration with the lead authors and two biomedical librarians, resulted in 7175 records (figure 1). Authors of this systematic review were asked to contribute any papers missed by the initial search, resulting in one addition. After removing duplicates, there were 5834 unique records. Two reviewers independently screened the titles of each record and excluded irrelevant studies or non-primary literature. 2293 records remained after this step. After applying exclusion criteria between two independent reviewers, 106 articles remained. The exclusion criteria were as follows: case reports, case series with $<5$ subjects, review articles, meta-analyses, editorials, animal research and research not pertaining to SRC. There were 
Table 1 Search string

Head injury and relevant sports.

This section will be combined with the searches below.

('Brain Injuries' [MESH] OR brain injur*[text word] OR concussion*[text word] OR postconcuss* [text word] OR commotio cerebri* [text word] OR post traumatic encephalopath*[text word] OR (post commotion[text word] OR post head injury[text word]) AND syndrome*[text word]) OR brain trauma*[text word] OR TBI[text word] OR MTBI[text word]) AND ('Athletes'[Mesh] OR 'Sports'[Mesh] OR sports[text word] OR sport[text s word] OR sporting[text word] OR athleti* [text word] OR athlete* [text word] OR 'recreation'[Mesh] OR recreat* [text word] OR baseball[text word] OR bicycling[text word] OR boxing[text word] OR cycling[text word] OR diving[text word] OR equestrian[text word] OR equine[text word] OR football[text word] OR 'Head Protective Devices'[Mesh] OR helmet ${ }^{\star}[$ text word] OR hockey[text word] OR lacrosse[text word] OR martial arts[text word] OR karate[text word] OR judo[text word] OR tae kwon do[text word] OR aikido[text word] OR mountaineering[text word] OR racquet sports[text word] OR tennis[text word] OR rugby[text word] OR skating[text word] OR skiing[text word] OR snow sports[text word] OR soccer[text word] OR wrestling[text word] OR 'Athletic Injuries'[Mesh))

Exercise tolerance
'Exercise Tolerance'(Mesh)
OR 'Heart Rate'(Mesh) OR
'Blood Pressure'(Mesh) OR
Exercise tolera*(text word)
OR Heart rate*(text word)
OR pulse rate*(text word)
OR blood pressure*(text
word)

Brain physiology

'Brain/physiology'(Mesh) OR

'Brain/physiopathology'(Mesh) OR

'Cerebrovascular Circulation'(Mesh)

OR 'Electrophysiology'(Mesh) OR

'Evoked Potentials'(Mesh) OR

brain physiolog*(text word) OR

brain pathophysiol*(text word) or

brain physiopathol* (text word) OR

Cerebral Circulation*(text word) OR

Cerebrovascular Circulation*(text word) OR Cerebral Blood Flow*(text word) OR electrophysio ${ }^{*}$ (text word) OR Event-related potential* (text word) OR evoked potential* ${ }^{*}$ text word)

\section{Advanced brain imaging}

'Magnetic Resonance Imaging'(Mesh) OR 'Magnetic Resonance Spectroscopy'(Mesh) OR 'Diffusion Tensor Imaging'(Mesh) OR 'PositronEmission Tomography'(Mesh) OR 'Tomography, Emission-Computed, Single-Photon'(Mesh) OR Neuroimaging(MeSH) OR MRI(text word) OR fMRI(text word) OR magnetic resonance imag*(text word) OR neuroimag*(text word) OR DTI(text word) OR Diffusion tensor imag*(text word) OR Diffusion Tractograph* (text word) OR ASL(text word) OR Arterial spin label* (text word) OR positron emission tomograph*(text word) OR PET(text word) OR single-photon emission computed tomograph*(text word) OR SPECT(text word)

Limits: English language, 1985-present, exclude animal only studies.

CBF, cerebral blood flow; DTI, diffusion tensor imaging; ERC, event-related potential; MRS, MS spectroscopy.

no discrepancies in applying the exclusion criteria. Six pairs of reviewers independently extracted relevant data, rated bias and level of evidence and removed an additional 26 papers that did not address the relevant questions, resulting in a final set of 80 studies. We used the Newcastle-Ottawa Scale to assess the risk of bias in included articles (using either the case-control or cohort version, depending on the article http:/www.ohri.ca/programs/ clinical_epidemiology/nosgen.pdf). Case series and cross-sectional studies were not ratable. Level of evidence was graded for each article with the Strength of Recommendation Taxonomy (SORT) (http://www.aafp.org/afp/2004/0201/p548.html). After the initial independent extraction and for all subsequent ratings of the level of evidence and risk of bias, each reviewer pair had to reconcile their ratings or a third 'tie-breaker' reviewer made the final determination.

A major challenge of this review is the broad range of modalities that were considered as potential physiological markers after concussion. As each modality has distinct measurement methods, biological significance and time course, it was not possible to combine all modalities into a single 'physiological' metric. The authorship group determined that dividing the studies into discrete categories would allow for the most succinct and useful information, which might then be stratified by the level of evidence and applied to the questions. Papers that included more than one physiological category were evaluated and rated separately for each category. The three questions to be addressed overlap in that they require comparison of physiological markers to a time course of clinical markers that are indirectly related to risk and recovery and, in the absence of a definitive answer to question 1 , the ability to delineate specific answers to questions 2 and 3 was extremely limited.

\section{RESULTS}

The categories of physiological markers for each modality were as follows: (1) functional MRI (fMRI), (2) diffusion tensor imaging (DTI)/diffusion MRI, (3) magnetic resonance spectroscopy
(MRS), (4) cerebral blood flow (CBF), (5) electrophysiology, (6) heart rate, (7) response to exercise, (8) fluid biomarkers and (9) transcranial magnetic stimulation. Although the number of studies varied between physiological categories, the overall level of evidence between categories was similar with almost all studies rated level 3. Thus, no single physiological marker category was clearly superior to the others. Each category will be summarised below, with a more detailed evidence table provided in the online supplementary materials. While most of the articles reviewed did not specify exact criteria for concussion diagnosis, in general, they did require clinical diagnosis by an experienced healthcare provider.

\section{FUNCTIONAL MRI}

There were 18 studies investigating fMRI after concussion. Signal changes in fMRI are postulated to represent altered brain activation but there is no clear consensus on the time frame for fMRI recovery after SRC. There were changes in cerebral activation ranging from 3 days to 23 months after clinical recovery and/or return-to-play (RTP). ${ }^{8-23}$ However, some fMRI changes only appear after symptoms are improving or even after resolution, suggesting more persistent network modifications may be separate phenomena from acute concussion physiology. ${ }^{24} 25$

Summary: Changes in fMRI activation vary based on brain regions of interest, different time points studied and analytical method. There is a relative dearth of studies monitoring patients until full physiological recovery. fMRI can be used to examine brain activation during specific tasks as well as for analysing connectivity at rest.

\section{DIFFUSION TENSOR IMAGING/DIFFUSION MRI}

Seven studies examining alterations in DTI after SRC were included in this review. These are inherently difficult to compare directly because of differences in study populations, methodology, time points and brain region of interest analysed. Among 


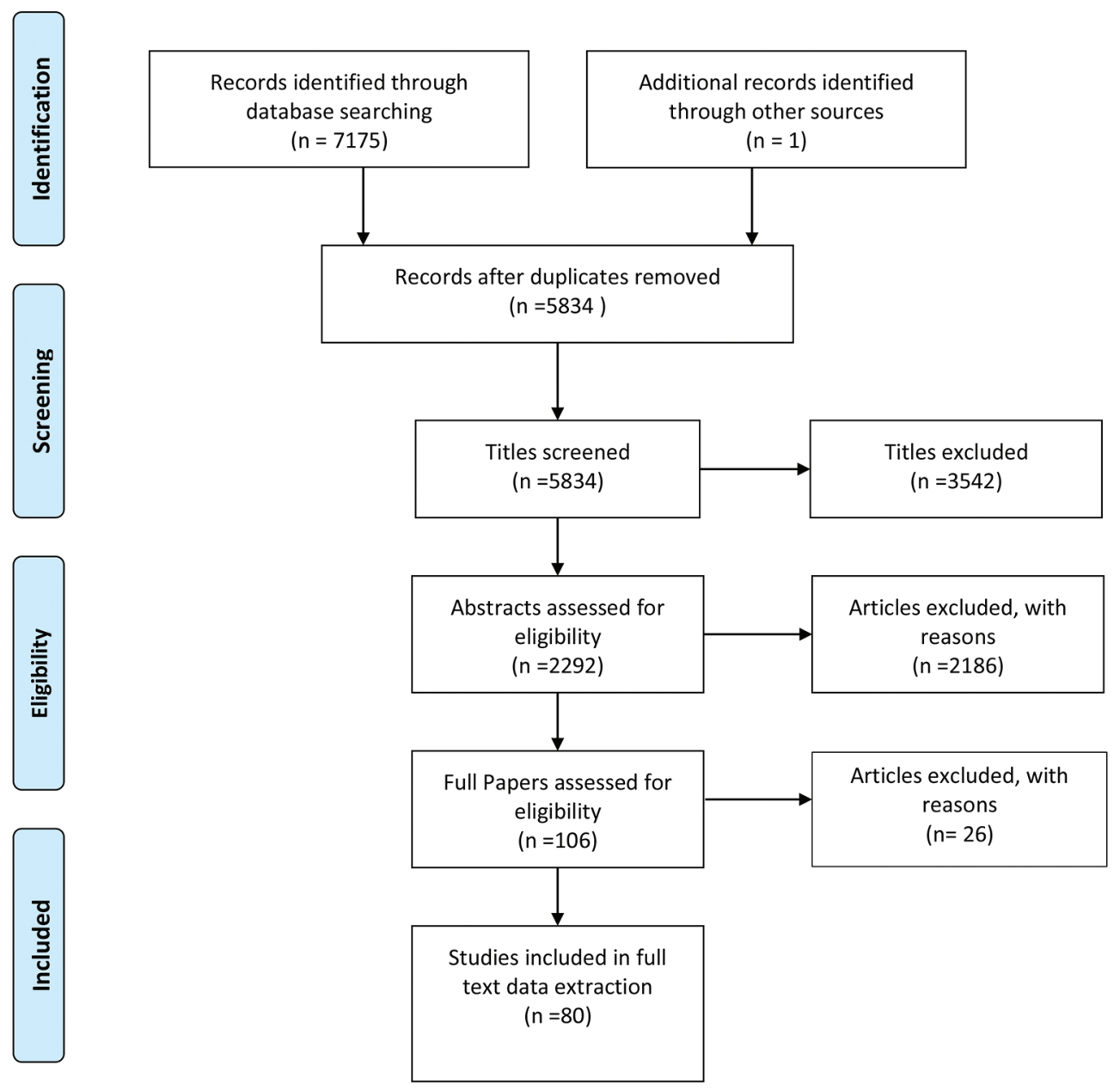

Figure 1 Literature search diagram

longitudinal studies, four used $\mathrm{DTI}^{26-29}$ and one used myelin water fraction (MWF). ${ }^{30}$ One article found imaging consistent with clinical data, with radial diffusivity increased and fractional anisotropy (FA) decreased at post injury day 2 (PID) with normalisation by 14 days after injury. ${ }^{26}$ One article examined both voxel and region of interest-based DTI analyses and reported increased FA at 1,8 and 32 days after injury. ${ }^{27}$ In this study, symptoms recovered between PID1-8 and behavioural surveys for anxiety and depression recovered between 8-32 days after injury. In one article, there was increased FA and axial diffusivity (AD) and decreased mean diffusivity (MD) that persisted from 5 to 180 days post injury. ${ }^{28}$ However, others found changes in FA varied depending on region of interest and showed increased MD at chronic time points. ${ }^{31}$ MWF was reduced at 3 days after injury, tended to be decreased at day 14 and normalised by day $60 .{ }^{30}$ Diffusion kurtosis imaging showed changes from controls at 1 day after injury and persisting at day 8 , but recovery of symptoms and cognition occurred between 1 and 8 days after injury. ${ }^{29}$

Summary: Following SRC, changes in white matter orientation as measured by DTI are detectable with different methods. Multiple, but not all, studies show persistence of these changes even as clinical symptoms and other measures (behavioural surveys, cognitive testing) tend to resolve over time. Thus, measures of white matter orientation are altered after SRC and may persist beyond clinical recovery. However, it is unclear what biological mechanisms underlie the DTI changes or whether similar changes in diffusivity represent different mechanisms at different time points.

\section{MAGNETIC RESONANCE SPECTROSCOPY}

There were a total of 10 studies that used magnetic resonance spectroscopy (MRS) to measure physiological change after sports concussion. Five studies demonstrated acute reductions in $\mathrm{N}$-acetylaspartate/creatine (NAA/Cr) ratios after SRC. Metabolic disturbance lasted until $7,,^{32} 15^{33}$ and up to $30^{313435}$ days after injury, despite recovery of clinical parameters by 15 days. ${ }^{3} 3637$ Another study showed more persistent alterations to the NAA/ Cr ratio. ${ }^{34}$ Three athletes who had a second concussion during the metabolic disturbance had prolonged NAA recovery. ${ }^{36}$ Abnormal ratios of myo-inositol or glutamate to creatine may be late but not acute markers of prior mild traumatic brain injury. ${ }^{31}$ 3538 One level 3 article showed abnormal gamma-NTP, a phosphorous metabolite, months after SRC. ${ }^{39}$

Summary: MRS can detect metabolic derangements following SRC. This evidence indicates that metabolic abnormalities may be detected beyond the window of clinical recovery. Preliminary evidence indicates that a repeat injury when the brain is metabolically vulnerable prolongs recovery. Additional work demonstrating the effect of repeat injury after clinical recovery, but before metabolic normalisation, will be required to determine whether metabolic recovery should be used to guide RTP recommendations.

\section{CEREBRAL BLOOD FLOW}

Phase contrast angiography, arterial spin labelling and transcranial Doppler have been assessed in eight articles. There was 
reduced cerebral blood flow $(\mathrm{CBF})$ at rest that persisted beyond the time of clinical recovery. Arterial spin labelling is an increasingly used, non-invasive technology that shows changes in CBF after concussion. ${ }^{40} \mathrm{CBF}$ abnormalities were resolved by 30 days post injury in most patients. ${ }^{41}{ }^{42}$ Although a single level 2 study demonstrated that global CBF was increased 40 days post injury in symptomatic athletes relative to controls, it decreased in asymptomatic athletes. ${ }^{43}$ Athletes may need to be challenged with exercise or hypercapnia to find this change in blood flow. ${ }^{22}$ ${ }^{44} 45$ There may be chronic effects on cerebrovascular reactivity that need to be further elucidated. ${ }^{46}$

Summary: Mounting evidence suggests an alteration of CBF following SRC, but the time course is not certain. Further longitudinal studies are needed to elucidate the acute versus chronic effects. The single study showing converse changes in blood flow depending on symptom level suggests that altered CBF may not definitively signify ongoing dysfunction.

\section{ELECTROPHYSIOLOGY}

Fifteen total studies used electrophysiological measures to assess concussion and recovery. The majority of electroencephalogram (EEG) and event-related potential (ERP) studies looked for chronic effects of concussion. Two studies, with unique EEG algorithms, demonstrated a significant injury effect at 8 days after concussion, with recovery by day $45,{ }^{47} 48$ although the athlete's symptoms recovered between days 5 and 8 . Eight studies showed persistent effects from 7 to 45 days after injury regardless of the symptom burden of the athletes ${ }^{249-55}$ Chronically, EEG and ERP demonstrate changes up to 4 years from injury that appear to outlast the clinical effects of concussion, with multiple studies showing decreased P300 amplitude on ERP. ${ }^{56-60}$

Summary: Various methods using ERP and several novel EEG algorithms have shown an electrophysiological effect of SRC. More longitudinal studies are needed to distinguish acute concussion physiology from remote effects of prior trauma. Two studies finding significant effects on EEG at 8 days post injury and recovery at 45 days after injury indicate possible physiological perturbations detectable after clinical recovery. However, given the proprietary algorithms that were used in these studies, replication will be needed before any definite conclusions can be made.

\section{HEART RATE}

Four articles (one level 2, three level 3) examined cardiac parameters after concussion. In one article, there was no significant change in resting heart rate or intervals, but a significant change in exercise-induced variability, a measure of sympathovagal balance ${ }^{61}$ In another article, there was shorter interbeat interval after concussion but a time course was not profiled. ${ }^{62}$ One study showed decreased complexity of heart rate during isometric hand grip testing on the second day after injury. ${ }^{63}$ In one article, there was decreased stroke volume (as estimated by SysSlope) on days 2 and 7 after injury. ${ }^{64}$

Summary: There is currently no specific modality or time course of cardiac function that delineates concussion recovery or correlates well with existing measures of clinical recovery.

\section{EXERCISE}

The role of controlled aerobic exercise to facilitate concussion recovery has provoked considerable interest, but the postinjury time course of impaired exercise tolerance has not been defined. Two studies demonstrated reduced exercise tolerance in subjects with chronic postconcussion syndrome. ${ }^{6566}$
Summary: There is insufficient evidence to delineate an accurate window of postinjury physiological dysfunction as related to exercise tolerance in the acute and subacute periods.

\section{FLUID (BLOOD AND URINE) BIOMARKERS}

Ten articles looked at potential fluid biomarkers for cerebral injury. ${ }^{67-76}$ Some examined correlates with clinical measures and others monitored trajectories of change after a diagnosed concussion. Cytoskeletal protein spectrin- $\mathrm{N}$-terminal-fragment was elevated at 12,36 and 144 hours after concussion $(n=18$ $25)$ and had returned to baseline in $(n=10)$ athletes who were clinically cleared for return to play. ${ }^{67}$ Total tau (another cytoskeletal molecule) was elevated after concussion, ${ }^{70}$ and if elevated at 144 hours after injury, was associated with persistent symptoms. Other markers (s100b, neuron-specific enolase) were not changed. ${ }^{68}$ Tau-C, but not Tau-A, was elevated after concussion; increased Tau-A at 144 hours was associated with persistent symptoms. ${ }^{69}$ S100b was increased 3 hours after concussion but not at days 2, 3, 7 or after exertion. ${ }^{71}$ Elevated glial fibrillar acidic protein was seen $<24$ hours postconcussion but not between 24 and 72 hours after injury; the change at $<24$ hours was associated with symptoms. ${ }^{72}$ One study examined biomarkers after impact exposure but not clinically diagnosed concussion, ${ }^{73}$ whereas others examined biomarkers after concussion but either did not project a time course of molecular change ${ }^{747}$ or looked at a broad set of markers. ${ }^{76}$

Summary: There are no reliable markers to monitor recovery from SRC. Fluid biomarkers have the potential to help inform either diagnosis or monitor for recovery after concussion. Before they may be recommended for clinical use, however, their utility must be demonstrated in longitudinal studies with limited attrition and clinical recovery as the primary outcome.

\section{TRANSCRANIAL MAGNETIC STIMULATION}

There were four level 3 studies that used transcranial magnetic stimulation. Three of these examined cortical excitability after concussion and found increased inhibition. One reported increased inhibition in the corticospinal tract at 2-4 days after concussion with normalisation by 10 days after injury, ${ }^{77}$ another found cortical hypo-excitability persisting beyond the time of clinical recovery, up to 28 days after injury, ${ }^{78}$ and the third article showed increased inhibition in the motor system that persisted beyond 9 months post injury. ${ }^{79}$ The last article took a different approach and reported prolonged motor latencies that persisted through PID10. ${ }^{80}$

Summary: In two articles, there were persistent abnormalities in cortical excitation beyond the window of clinical recovery. Another study found electrophysiological dysfunction acutely after SRC that did not correlate with symptoms. Further experiments are needed to define the time window of altered cortical excitability after SRC.

\section{DISCUSSION}

This article presents a wide range of data relating to physiological recovery after SRC. Much of the data suggest that the window for physiological recovery extends beyond that for recovery of clinical signs and symptoms. However, multiple considerations preclude making strong clinically actionable recommendations given the current evidence. These considerations fall into three main categories:

1. There is a broad range of different physiological measures studied after SRC, each of which almost certainly has a different time course, severity, method of measurement and 
clinical significance. These factors make it impossible to produce a single 'physiological time-window for concussion recovery' metric.

2. The real-world significance of each physiological modality should be delineated by its relationship to existing clinical parameters. The first step is to better determine the relationship between pathophysiology and known clinical symptoms or signs of neurological dysfunction. Understanding connections between physiological parameters and increased vulnerability to repeat concussion or to more severe acute/subacute symptoms is important for clinical management of return to activity/return to play. Correlation of pathophysiology to longer term risk of chronic or degenerative problems is more difficult and requires prospective, longitudinal study designs with extended follow-up.

3. The literature reviewed here reflects evidence directed at showing biological changes after SRC. It is possible that measurable brain changes occur after repeated brain impacts in the absence of clinically diagnosed SRC, sometimes termed 'subconcussion'. Whether these brain changes confer resiliency, vulnerability or have no clinically measureable effect remains to be seen. However, by focusing on clinically diagnosed concussions in this review, the physiological changes and their time courses summarised here may or may not be relevant in the sports setting where repeated impacts also occur without recognition or diagnosis of a concussion.

This review clearly revealed major limitations to the existing body of evidence relating to pathophysiological recovery after SRC. The most glaring study design limitation is a scarcity of longitudinal cohort studies that follow patients until resolution of the physiological dysfunction and correlated it to clinical recovery measures. This is crucial to find the duration of clinically relevant neurobiological dysfunction. Most included articles were cross-sectional design (with single time points) or cohort studies that did not follow subjects to full recovery. Where possible, analysis of the consequences of repeat injury occurring before normalisation of physiological changes may be enlightening if a linkage can be established between repeat injury, pathophysiology and clinical dysfunction. Such studies may be difficult and require large observational cohorts. However, it is possible to examine pathophysiological risk using preclinical animal models.

The generalisability of individual studies with small sample sizes is limited, given frequent selection biases, unique study populations and inappropriate or absent controls. There is a common theme of finding significant results using very sensitive methods with uncertain clinical significance. These studies are prone to bias, particularly with computational analyses of advanced imaging or electrophysiology, and the results should be interpreted cautiously.

All physiological studies are hindered by the absence of a gold-standard clinical definition of concussion and thus are threatened by measurement bias. This is heightened by the lack of consensus on what is deemed 'recovery', especially in the light of ongoing chronic premorbid symptoms such as chronic migraine or mood disorders.

Methodologically, this systematic review is subject to publication and language bias as we reviewed only articles published in English.

\section{SUMMARY AND RECOMMENDATIONS}

The review aimed to address the following three questions:
1. How does the time course of physiological recovery compare with the time line of clinical recovery after SRC?

2. Should there be a minimum stand-down period following SRC?

3. Is the duration or content of graded return to play progressions appropriate?

\section{Recommendation 1}

How does the time course of physiological recovery compare with the time line of clinical recovery after SRC?

The time course of recovery for multiple physiologically measurable parameters appears to exceed clinical recovery. A definitive time point for physiological recovery after SRC is yet to be determined. Reliable studies of MRS and CBF appear to demonstrate a physiological disturbance for greater than 15 days but less than 30 days after injury. This is not only longer than the average clinical time to recovery in adults, but in individual papers there was evidence of clinical recovery prior to physiological recovery. ${ }^{363740}$ The time course of biological disturbance is less clear with other modalities like DTI, electrophysiology and fluid biomarkers. Longitudinal studies are necessary to characterise the progression of physiological recovery. It is unclear whether changes in neurobiology represent dysfunction, vulnerability or recovery.

It is prudent to incorporate a 'buffer zone' of gradually increasing cognitive and physical activity between the time of neurological (symptomatic) recovery and return to full contact risk.

\section{Recommendation 2}

Should there be a minimum stand-down period following SRC?

There is limited clinical evidence that repeat concussion during the window of neurometabolic disturbance leads to prolonged recovery. Therefore, from a physiological standpoint, we are unable to determine whether there needs to be a minimum standdown period after injury. The most rigorous evidence regarding this still comes from animal data where intersubject variability is low, injury severity consistent and time points are precise. These studies show vulnerability ${ }^{8182}$ if a repeat injury occurs before complete metabolic recovery. The duration of this time window in humans with concussion is unclear. The significance of 'subconcussive impacts' and biological changes reported after playing contact sports for a set time are also unclear. Animal and human studies indicate that repeat concussion prior to recovery result in more severe and prolonged physiological and clinical disturbances. This supports immediate removal from contact risk after an identified injury.

As there is (are) currently no standard biomarker(s) for physiological dysfunction underlying vulnerability to repeat concussion in humans, the stand-down time should be determined by thorough clinical assessment.

\section{Recommendation 3}

Is the duration or content of graded return to play progressions appropriate?

There is insufficient evidence to recommend a change to the current graded return to play progression. To answer this question, there is need for data on the effect of repeat brain injury and the effect of exercise on ongoing concussion physiology. These studies need to be adequately powered, have a longitudinal component, use one or more potential clinical correlates and have appropriate controls. Such studies are necessary to determine the time course of both physiological and clinically 
measurable dysfunction. Longitudinal analyses are important in determining whether subclinical changes become clinically relevant with ageing or later developing disease processes. Outcomes from such studies need to be carefully defined with respect to risk of repeat injury, risk of new musculoskeletal injury, risk of worsened symptoms, prolongation of recovery, neurocognitive impairment or chronic neurodegeneration.

Further studies investigating physiological biomarkers of concussion should be undertaken. These studies should select generalisable populations, implementing longitudinal cohorts that follow patients to physiological and clinical recovery and examine the relationship between physiological disturbance and clinical outcomes. While not specific to this question, our understanding of concussion physiology would certainly benefit from more objective diagnostic and recovery criteria, and research to better define 'concussion' and 'recovery' is a high priority.

\section{CONCLUSION}

Given the limitations of the available data, we recommend that medical providers continue to use clinical data to manage return to play while reserving physiological metrics for research until more conclusive data are obtained. This review shows that the window of physiological dysfunction can outlast that of clinical recovery. These data support using a 'buffer zone' of a graduated return to activity/return to play progression before full return to contact risk.

\author{
Author affiliations \\ 'Department of Neurosurgery, UCLA Steve Tisch BrainSPORT Program, Los Angeles, \\ California, USA \\ 2UCLA Brain Injury Research Center, Los Angeles, California, USA \\ ${ }^{3}$ Department of Psychology and Neuroscience Center, Brigham Young University, Salt \\ Lake City, Utah, USA \\ ${ }^{4}$ Michigan State University, East Lansing, Michigan, USA \\ ${ }^{5}$ Department of Neurosurgery, University of Pittsburgh, Pittsburgh, Pennsylvania, \\ United States \\ ${ }^{6}$ Sports Medicine, Rugby Football Union, London, UK \\ ${ }^{7}$ Orthopaedics, SUNY Buffalo, Buffalo, New York, USA \\ ${ }^{8}$ The Mind Research Network, University of New Mexico, Albuquerque, New Mexico, USA \\ ${ }^{9}$ Department of Neurosurgery, Medical College of Wisconsin, Milwaukee, Wisconsin, USA \\ ${ }^{10}$ Department of Neurosurgery, UCLA Brain Injury Research Center, Los Angeles, \\ California, USA \\ ${ }^{11}$ Faculty of Kinesiology, University of Calgary, Calgary, Canada \\ ${ }^{12}$ Interdisciplinary Health Sciences, A.T. Still University, Mesa, Arizona, USA \\ ${ }^{13}$ Department of Pediatrics, Children's Hospital of Eastern Ontario, Ottawa, Canada \\ ${ }^{14}$ Department of Pediatrics/Pediatric Neurology, Mattel Children's Hospital UCLA, Los \\ Angeles, California, USA
}

Competing interests JK-Fellowship partially supported by UCLA Steve Tisch BrainSPORT programme. EB - grants/research support: National Institute of Child Health and Human Development (grant Nos 5R01HD048946 and 3R01HD04894605S1) and the USA. Army Medical Research and Material Command under Award No W81XWH-13-2-0095 (Chronic Effects of Neurotrauma Consortium); DirectorNeuropsychological Research and Assessment Lab at BYU's Comprehensive Clinic. Medicolegal: Some forensic cases. TC — grants/research support: Brainscope, Datalys, Pentecost Foundation. SK—Chief Medical Officer, Rugby Football Union; Committee Member: World Rugby Head Injury Assessment Working Group and Football Association Independent Panel on Concussion and Head Injury. JJL_grants/ research support: NIH, The Ralph C. Wilson Foundation, Program for Understanding Childhood Concussion and Stroke, The Robert Rich Family Foundation and The Buffalo Sabres Foundation. AM—grants/research support: NIH, US Dept of Defense, NSF. MMC — grants/research support: US Dept of Defense, US Dept of Veteran's Affairs, NCAA, NFL-GE, GE Health Care, MCW CTSI, private donors; Consultant: Green Bay Packers; Past member: NFL Head, Neck and Spine Committee (w/o compensation): Member: GE Healthcare Medical Advisory Board, Board of Governors for International Neuropsychological Society. KJS — Consultant, Evidence Sport and Spinal Therapy; speaking honoraria for presentations at scientific meetings. TCVM-Trustee, United States Brain Injury Alliance; Senior Associate Editor: Journal of Athletic Training, Associate Editor: Journal of Sport Rehabilitation; Consultant, Concussion Health. RZ - grants/research support: CIHR, Ontario Neurotrauma Foundation, Ontario SPOR
Support Unit, Clinical Research Chair in Pediatric Concussion, University of Ottawa Brain and Mind Institute. CCG—grants/research support: NIH, NCAA, US Department of Defense, Today's and Tomorrow's Children Fund, UCLA Brain Injury Research Center, UCLA Faculty Grants Program, UCLA Steve Tisch BrainSPORT programme, Avanir (research grant 2017-2018), NINDS Neural Analytics SBIR grant (2016-2018); Consultant: NFL-Neurological Care Program, NHLPA, Neural Analytics Inc (2015-16); Advisory Panel: LoveYourBrain, MLS, NBA, NCAA, USSF; Medicolegal: One or two cases annually; Speaker's Bureau: Medical Education Speakers Network.

Provenance and peer review Not commissioned; externally peer reviewed.

(c) Article author(s) (or their employer(s) unless otherwise stated in the text of the article) 2017. All rights reserved. No commercial use is permitted unless otherwise expressly granted.

\section{REFERENCES}

1 Meier TB, Bellgowan PS, Singh R, et al. Recovery of cerebral blood flow following sports-related concussion. JAMA Neurol 2015;72:530-8.

2 Prichep LS, McCrea M, Barr W, et al. Time course of clinical and electrophysiological recovery after sport-related concussion. J Head Trauma Rehabil 2013;28:266-73.

3 Vagnozzi R, Signoretti S, Floris R, et al. Decrease in $\mathrm{N}$-acetylaspartate following concussion may be coupled to decrease in creatine. J Head Trauma Rehabil 2013;28:284-92

4 Guskiewicz KM, Mccrea M, Marshall SW, et al. The NCAA concussion study. J Am Med Assoc 2003;290:2549-55.

5 McKee AC, Stern RA, Nowinski CJ, et al. The spectrum of disease in chronic traumatic encephalopathy. Brain 2013;136:43-64.

6 Prins ML, Alexander D, Giza CC, et al. Repeated mild traumatic brain injury: mechanisms of cerebral vulnerability. J Neurotrauma 2013;30:30-8.

7 Cross M, Kemp S, Smith A, et al. Professional rugby union players have a $60 \%$ greater risk of time loss injury after concussion: a 2-season prospective study of clinical outcomes. Br J Sports Med 2016;50:926-31.

8 Borich M, Babul AN, Yuan PH, et al. Alterations in resting-state brain networks in concussed adolescent athletes. J Neurotrauma 2015;32:265-71.

9 Chen JK, Johnston KM, Frey S, et al. Functional abnormalities in symptomatic concussed athletes: an fMRI study. Neuroimage 2004;22:68-82.

10 Chen JK, Johnston KM, Collie A, et al. A validation of the post concussion symptom scale in the assessment of complex concussion using cognitive testing and functional MRI. J Neurol Neurosurg Psychiatry 2007;78:1231-8.

11 Chen JK, Johnston KM, Petrides M, et al. Recovery from mild head injury in sports: evidence from serial functional magnetic resonance imaging studies in male Athletes. Clin J Sport Med 2008;18:241-7.

12 Czerniak SM, Sikoglu EM, Liso Navarro AA, et al. HHS public access. Brain Imaging Behav 2014;67:223-30.

13 Dettwiler A, Murugavel M, Putukian M, et al. Persistent differences in patterns of brain activation after sports-related concussion: a longitudinal functional magnetic resonance imaging study. J Neurotrauma 2014;31:180-8.

14 Elbin RJ, Covassin T, Hakun J, et al. Do brain activation changes persist in athletes with a history of multiple concussions who are asymptomatic? Brain Inj 2012;26:1217-25.

15 Hammeke TA, McCrea M, Coats SM, et al. Acute and subacute changes in neural activation during the recovery from sport-related concussion. J Int Neuropsycho/ Soc 2013;19:863-72

16 Johnson B, Zhang K, Gay M, et al. Alteration of brain default network in subacute phase of injury in concussed individuals: resting-state fMRI study. Neuroimage 2012:59:511-8

17 Johnson B, Neuberger T, Gay M, et al. Effects of subconcussive head trauma on the default mode network of the brain. J Neurotrauma 2014;31:1907-13.

18 Keightley ML, Saluja RS, Chen JK, et al. A functional magnetic resonance imaging study of working memory in youth after sports-related concussion: is it still working? J Neurotrauma 2014;31:437-51.

19 Leddy JJ, Cox JL, Baker JG, et al. Exercise treatment for postconcussion syndrome: a pilot study of changes in functional magnetic resonance imaging activation, physiology, and symptoms. J Head Trauma Rehabil 2013;28:249-241.

20 Lovell MR, Pardini JE, Welling J, et al. Functional brain abnormalities are related to clinical recovery and time to return-to-play in athletes. Neurosurgery 2007;61:352-60

21 Militana AR, Donahue MJ, Sills AK, et al. Alterations in default-mode network connectivity may be influenced by cerebrovascular changes within 1 week of sports related concussion in college varsity athletes: a pilot study. Brain Imaging Behav 2016:10:559-68.

22 Mutch WA, Ellis MJ, Ryner LN, et al. Brain magnetic resonance imaging CO2 stress testing in adolescent postconcussion syndrome. J Neurosurg 2016;125:1-13.

23 Talavage TM, Nauman EA, Breedlove EL, et al. Functionally-detected cognitive impairment in high school football players without clinically-diagnosed concussion. J Neurotrauma 2014;31:327-38. 
24 Meier TB, Bellgowan PS, Mayer AR. Longitudinal assessment of local and global functional connectivity following sports-related concussion. Brain Imaging Behav 2016 (Published Online First).

25 Zhu DC, Covassin T, Nogle S, et al. A potential biomarker in sports-related concussion: brain functional connectivity alteration of the default-mode network measured with longitudinal resting-state fMRI over thirty days. J Neurotrauma 2015;32:327-41.

26 Murugavel M, Cubon V, Putukian M, et al. A longitudinal diffusion tensor imaging study assessing white matter fiber tracts after sports-related concussion. J Neurotrauma 2014;31:1860-71.

27 Meier TB, Bergamino M, Bellgowan PS, et al. Longitudinal assessment of white matter abnormalities following sports-related concussion. Hum Brain Mapp 2016;37:833-45.

28 Henry LC, Tremblay J, Tremblay S, et al. Acute and chronic changes in diffusivity measures after sports concussion. J Neurotrauma 2011;28:2049-59.

29 Lancaster MA, Olson DV, McCrea MA, et al. Acute white matter changes following sport-related concussion: a serial diffusion tensor and diffusion kurtosis tensor imaging study. Hum Brain Mapp 2016;37:3821-34.

30 Wright $A D$, Jarrett $M$, Vavasour I, et al. Myelin water fraction is transiently reduced after a single mild traumatic brain injury--A prospective cohort study in collegiate hockey players. PLoS One 2016;11:e0150215.

31 Chamard E, Lassonde M, Henry L, et al. Neurometabolic and microstructural alterations following a sports-related concussion in female athletes. Brain Inj 2013:27:1038-46.

32 Henry LC, Tremblay S, Boulanger Y, et al. Neurometabolic changes in the acute phase after sports concussions correlate with symptom severity. J Neurotrauma 2010;27:65-76.

33 Johnson B, Gay M, Zhang K, et al. The use of magnetic resonance spectroscopy in the subacute evaluation of athletes recovering from single and multiple mild traumatic brain injury. J Neurotrauma 2012;29:2297-304.

34 Henry LC, Tremblay S, Leclerc $S$, et al. Metabolic changes in concussed American football players during the acute and chronic post-injury phases. BMC Neurol 2011;11:105.

35 Chamard E, Henry L, Boulanger Y, et al. A follow-up study of neurometabolic alterations in female concussed athletes. J Neurotrauma 2014;31:339-45.

36 Vagnozzi R, Signoretti S, Tavazzi B, et al. Temporal window of metabolic brain vulnerability to concussion: a pilot $1 \mathrm{H}$-Magnetic resonance spectroscopic study in concussed Athletes-Part III. Neurosurgery 2008;62:1286-96.

37 Vagnozzi R, Signoretti S, Cristofori L, et al. Assessment of metabolic brain damage and recovery following mild traumatic brain injury: a multicentre, proton magnetic resonance spectroscopic study in concussed patients. Brain 2010;133:3232-42.

38 Chamard E, Théoret H, Skopelja EN, et al. A prospective study of physician-observed concussion during a varsity university hockey season: metabolic changes in ice hockey players. part 4 of 4. Neurosurg Focus 2012;33:E4.

39 Sikoglu EM, Liso Navarro AA, Czerniak SM, et al. Effects of recent concussion on brain bioenergetics: a Phosphorus-31 magnetic resonance spectroscopy study. Cogn Behav Neuro/ 2015;28:181-7.

40 Wang Y, Nelson LD, LaRoche AA, et al. Cerebral blood flow alterations in acute sportrelated concussion. J Neurotrauma 2016;33:1227-36.

41 Meier TB, Bellgowan PS, Singh R, et al. Recovery of cerebral blood flow following sports-related concussion. JAMA Neurol 2015;72:530-8.

42 Maugans TA, Farley C, Altaye M, et al. Pediatric sports-related concussion produces cerebral blood flow alterations. Pediatrics 2012;129:28-37.

43 Barlow KM, Marcil LD, Dewey D, et al. Cerebral perfusion changes in post-concussion syndrome: a prospective controlled cohort study. J Neurotrauma 2017:34:996-1004.

44 Len TK, Neary JP, Asmundson GJ, et al. Serial monitoring of CO2 reactivity following sport concussion using hypocapnia and hypercapnia. Brain Inj 2013;27:346-53.

45 Clausen M, Pendergast DR, Willer B, et al. Cerebral blood flow during treadmill exercise is a marker of physiological postconcussion syndrome in female Athletes. $J$ Head Trauma Rehabil 2016;31:215-24.

46 da Costa L, van Niftrik CB, Crane D, et al. Temporal profile of cerebrovascular reactivity impairment, gray matter volumes, and persistent symptoms after mild traumatic head injury. Front Neurol 2016;7:70.

47 Barr WB, Prichep LS, Chabot R, et al. Measuring brain electrical activity to track recovery from sport-related concussion. Brain Inj 2012;26:58-66.

48 McCrea M, Prichep L, Powell MR, et al. Acute effects and recovery after sport-related concussion: a neurocognitive and quantitative brain electrical activity study. $J$ Head Trauma Rehabil 2010;25:283-92.

49 Cao C, Tutwiler RL, Slobounov S. Automatic classification of athletes with residual functional deficits following concussion by means of EEG signal using support vector machine. IEEE Trans Neural Syst Rehabil Eng 2008;16:327-35.

50 Cao C, Slobounov S. Alteration of cortical functional connectivity as a result of traumatic brain injury revealed by graph theory, ICA, and sLORETA analyses of EEG signals. IEEE Trans Neural Syst Rehabil Eng 2010;18:11-19.

51 Cao C, Slobounov S. Application of a novel measure of EEG non-stationarity as 'Shannon- entropy of the peak frequency shifting' for detecting residual abnormalities in concussed individuals. Clin Neurophysiol 2011:122:1314-21.
52 Gosselin N, Thériault M, Leclerc S, et al. Neurophysiological anomalies in symptomatic and asymptomatic concussed Athletes. Neurosurgery 2006;58:1151-61.

53 Slobounov S, Sebastianelli W, Moss R. Alteration of posture-related cortical potentials in mild traumatic brain injury. Neurosci Lett 2005;383:251-5.

54 Slobounov S, Cao C, Sebastianelli W. Differential effect of first versus second concussive episodes on wavelet information quality of EEG. Clin Neurophysiol 2009:120:862-7.

55 Slobounov S, Sebastianelli W, Hallett M. Residual brain dysfunction observed one year post-mild traumatic brain injury: combined EEG and balance study. Clin Neurophysio/ 2012;123:1755-61.

56 Broglio SP, Pontifex MB, O'Connor $\mathrm{P}$, et al. The persistent effects of concussion on neuroelectric indices of attention. J Neurotrauma 2009;26:1463-70.

57 Baillargeon A, Lassonde M, Leclerc S, et al. Neuropsychological and neurophysiological assessment of sport concussion in children, adolescents and adults. Brain Inj 2012;26:211-20.

58 Gosselin N, Bottari C, Chen J-K, et al. Evaluating the cognitive consequences of mild traumatic brain injury and concussion by using electrophysiology. Neurosurg Focus 2012:33:E7.

59 Lavoie ME, Dupuis F, Johnston KM, et al. Visual p300 effects beyond symptoms in concussed college athletes. J Clin Exp Neuropsychol 2004;26:55-73.

60 Dupuis F, Johnston KM, Lavoie M, et al. Concussions in Athletes produce brain dysfunction as revealed by event-related potentials. Neuroreport 2000;11:4087-92.

61 Abaji JP, Curnier D, Moore RD, et al. Persisting effects of concussion on heart rate variability during physical exertion. J Neurotrauma 2016;33:811-7.

62 Gall B, Parkhouse W, Goodman D. Heart rate variability of recently concussed athletes at rest and exercise. Med Sci Sports Exerc 2004;36:1269-74.

63 La Fountaine MF, Heffernan KS, Gossett JD, et al. Transient suppression of heart rate complexity in concussed athletes. Auton Neurosci 2009;148:101-3.

64 La Fountaine MF, Toda M, Testa AJ, et al. Autonomic nervous system responses to concussion: arterial pulse contour analysis. Front Neurol 2016;7:13.

65 Kozlowski KF, Graham J, Leddy JJ, et al. Exercise intolerance in individuals with postconcussion syndrome. J Ath/ Train 2013;48:627-35.

66 Leddy JJ, Baker JG, Kozlowski K, et al. Reliability of a graded exercise test for assessing recovery from concussion. Clin J Sport Med 2011;21:89-94.

67 Siman R, Shahim P, Tegner Y, et al. Serum SNTF increases in concussed professional Ice Hockey players and relates to the severity of postconcussion symptoms. J Neurotrauma 2015;32:1294-300.

68 Shahim $P$, Linemann $T$, Inekci $D$, et al. Serum tau fragments predict return to play in concussed professional ice hockey players. J Neurotrauma 2016;33:1995-9.

69 Shahim $\mathrm{P}$, Tegner $Y$, Wilson DH, et al. Blood biomarkers for brain injury in concussed professional ice hockey players. JAMA Neuro/ 2014;71:684-92.

70 Neselius S, Zetterberg H, Blennow K, et al. Olympic boxing is associated with elevated levels of the neuronal protein tau in plasma. Brain Inj 2013;27:425-33.

71 Kiechle K, Bazarian JJ, Merchant-Borna K, et al. Subject-specific increases in serum S-100B distinguish sports-related concussion from sports-related exertion. PLoS One 2014;9:e84977.

72 Mannix R, Eisenberg M, Berry M, et al. Serum biomarkers predict acute symptom burden in children after concussion: a preliminary study. J Neurotrauma 2014;31:1072-5.

73 Marchi N, Bazarian JJ, Puvenna V, et al. Consequences of repeated blood-brain barrier disruption in football players. PLoS One 2013;8:e56805.

74 Dambinova SA, Shikuev AV, Weissman JD, et al. AMPAR peptide values in blood of nonathletes and club sport athletes with concussions. Mil Med 2013;178:285-90.

75 Oliver J, Abbas K, Lightfoot JT, et al. Comparison of neurocognitive testing and the measurement of marinobufagenin in mild traumatic brain injury: a preliminary report. J Exp Neurosci 2015;9:67-72.

76 Gill J, Merchant-Borna K, Lee H, et al. Sports-Related concussion results in differential expression of nuclear Factor-kappaB pathway genes in peripheral blood during the acute and subacute periods. I Head Trauma Rehabil 2015

77 Pearce AJ, Hoy K, Rogers MA, et al. Acute motor, neurocognitive and neurophysiological change following concussion injury in Australian amateur football. A prospective multimodal investigation. J Sci Med Sport 2015;18:500-6.

78 Powers KC, Cinelli ME, Kalmar JM. Cortical hypoexcitability persists beyond the symptomatic phase of a concussion. Brain Inj 2014;28:465-71.

79 De Beaumont L, Mongeon D, Tremblay S, et al. Persistent motor system abnormalities in formerly concussed athletes. J Athl Train 2011;46:234-40.

80 Livingston SC, Goodkin HP, Hertel JN, et al. Differential rates of recovery after acute sport-related concussion: electrophysiologic, symptomatic, and neurocognitive indices. J Clin Neurophysio/ 2012;29:23-32.

81 Longhi L, Saatman KE, Fujimoto S, et al. Temporal window of vulnerability to repetitive experimental concussive brain injury. Neurosurgery 2005:56:364-74

82 Prins ML, Hales A, Reger M, et al. Repeat traumatic brain injury in the juvenile rat is associated with increased axonal injury and cognitive impairments. Dev Neurosci 2011;32:510-8 